\title{
Research in Prehospital and Disaster Health and Medicine: Developing a Research Objective Statement
}

\author{
Samuel J. Stratton, MD, MPH
}

In the previous issue of Prehospital and Disaster Medicine it was reported that a common flaw in manuscripts submitted to the Journal is lack of an organized and concise statement of the objective for a study. ${ }^{1}$ Much is written about study design and statistical analysis techniques, but little is written regarding the basis for a scientific study: the research objective statement or hypothesis. This essential element of a research project helps determine the study methodology and defines the study population, interventions, and possible outcome of interest. A carefully defined and concisely stated research objective determines the focus of a study and helps those reading a report of the research concentrate on what the research is about. A flawed research objective statement leads to confusion for those evaluating a study and often leads to researcher muddling in conduct of the research.

\section{Objective of this Monograph}

This editorial monograph explores the development of a research objective statement as it applies to prehospital and disaster research methods. Approaches for developing a research objective are explored, with recommendations made for accomplishing a concise and well-focused objective (hypothesis) statement. Quantitative and qualitative studies are the types of research methodologies discussed.

\section{Forming a Research Objective Statement}

Interest in a research project often originates from a number of sources, including the personal interests of the researcher, unanswered questions that arise from current research, and concern about current controversy or new interventions; frequently topics are generated by study grant proposals or other researchers.

For an objective to be worth the time to study, it must first pass the "who cares?" test. "Who cares?" is a euphemism used to express the idea that a topic or statement is not of interest or importance. A potential research objective is important and of interest if it addresses an area of clinical importance for which there is controversy or a gap in the knowledge base.

Not only is developing a new research objective a means to developing a study, it is also appropriate to repeat a study using the original research question if there is interest and a need to confirm the findings of the original research. Repeating controversial studies is a mechanism for validation of the original findings. A basis for science is that the results of valid research are reproducible.

In addition to recognizing a gap or controversy for the knowledge that exists, it is important that the current literature for a potential research topic be known in order to develop a plausible research objective. This is necessary when a substantial

amount of research and evaluation has been done on a particular topic. If knowledge of a topic has developed beyond a basic level, then research of the topic at that basic level will add little to the knowledge base and be of little interest to others. Also, knowing the background and literature for a topic helps to support the need for a proposed research objective.

\section{Structure of a Research Objective Statement}

A research objective statement should be concise and express the full intent of the research. Preferably, a research objective is stated within a single sentence. Many studies have a general research objective with secondary objectives. Each of these objectives should be concise and stated separately. To avoid over-extending a single study, as few research objectives as are necessary to explore an important gap in knowledge should be proposed. Therefore, a single concise statement of the research objective is preferred as opposed to multiple objectives because each additional objective distracts from the primary objective and tends to lead to poorly-focused and confused research.

To explore the structure of a workable research objective, it is necessary to define the following terms:

Variable - a measure or condition that may vary or change Independent variable - a variable that changes and has an effect on the outcome of interest

Dependent variable - a variable that is determined by the effects of independent variables (the outcome).

In simple terms, a classic research objective is expressed using the following:

$$
\begin{gathered}
\text { Independent Variable \# } 1+/- \text { Independent Variable \# } 2 \\
=\text { Dependent Variable }
\end{gathered}
$$

In prehospital and disaster health and medical research, independent variable \# 1 is most often a defined community or population and occasionally a resource (such as hospital availability). Independent variable \# 2 is usually an intervention or event that is believed to have an effect on the community or resource, and the dependent variable is the outcome of interest for the research. Therefore, in functional terms, a basic study objective for prehospital and disaster medical and health research can be stated as:

$$
\begin{aligned}
& \text { Population or Resource of Interest }+/- \text { Intervention (or event) } \\
& =\text { Outcome }
\end{aligned}
$$

While the above structure for a research objective is simple, the application of the concept varies depending on the intended 
method for conducting a study. For example, consider a research question in which it is desired to determine how much water for oral intake is required for survivors of an earthquake at an evacuation site. The research objective first must be framed by defining the community of interest. For this example, assume the community (study population) is the families evacuated into a shelter because of an earthquake that occurred in a temperate zone during summer months. The intervention for the study objective is the amount of oral intake water available which can be measured quantitatively or subjectively. For purposes of this example, assume the desired outcome measure is control of thirst. Also assume that prior literature on the topic has recommended that a minimum of three liters of water per 24-hour period be available for oral intake per person in general day to day situations. This information allows for developing study objectives in different ways that fit various study methods:

Example study objective for a quantitative study:

For families in an earthquake evacuation center in a temperate climate during summer, three liters of water for oral intake per person during a 24-hour period will result in control of thirst.

Example study objective for a qualitative study:

This study of families in an earthquake evacuation center during summer months in a temperate climate explores how much water for oral intake per person during a 24-hour period controls thirst.

Note that the two types of study approach the research objective in ways unique to each method, but that the research objective is stated for each method such that a similar answer to the question should be obtained.

In addition to the basic study objective, comparisons or multiple independent variables can be used in developing a study objective. In this situation, the basic structure for a study objective changes to:

$$
\begin{aligned}
& \text { Population or Resource of Interest }+/ \text { - Interventions (or events) } \\
& =\text { Outcome }
\end{aligned}
$$

Or if written using the example of water for oral intake in an evacuation center:

For families in an earthquake evacuation center in a temperate climate during summer, this study compared the effectiveness of 3 liters compared to 2 liters of water for oral intake per person during a 24-hour period in controlling thirst.

In each of the written study objective examples, a time qualifier is included. When time intervals or periods will help define a study variable, that time element should be included in the research objective statement.

Often, simple descriptive studies are used to define populations or resources. These types of studies are valuable for prehospital and disaster research because they provide a baseline measure for future comparisons and allow for understanding the structure of a population or resource that may be affected by an event or intervention. A study objective for a descriptive study still follows the format described above; for example, if one wished to describe the demographics or characteristics of the

\begin{tabular}{|c|c|}
\hline $\mathbf{P}$ & Population = study population or study resource of interest \\
\hline I & $\begin{array}{c}\text { Intervention = event, action, evaluation, or change applied to } \\
\text { the study population or resource }\end{array}$ \\
\hline C & $\begin{array}{c}\text { Comparison Group = comparison population, control group, } \\
\text { or published comparison statistics }\end{array}$ \\
\hline O & $\begin{array}{c}\text { Outcome of Interest = expected result of intervention on study } \\
\text { population or resource }\end{array}$ \\
\hline $\mathbf{T}$ & $\begin{array}{c}\text { Time = time of occurrence, time length of intervention, time } \\
\text { unit of measure, follow-up time }\end{array}$ \\
\hline
\end{tabular}

Table 1. The PICOT Criteria Checklist of the Essential Elements of a Well-developed Research Objective Statement ${ }^{2}$

population of an evacuation center, the study objective could be written:

This study determined the population characteristics of families in an earthquake evacuation center in a temperate climate during summer.

To better focus the descriptive study objective above, the population characteristics evaluated and timing of the study should be stated in a second sentence, such as:

This study determined the population characteristics of families in an earthquake evacuation center in a temperate climate during summer. The study was conducted the first three days after the July, 2014 event, and characteristics evaluated were age, gender, marital status, parental status, known pregnancy status, disabilities, and current use of prescribed medications.

Disaster event dynamics change with time and temporal changes can affect descriptive studies. Time is also an element for prehospital studies.

\section{The Research Objective Statement Drives a Study}

The research objective should be developed before a study begins and data is collected. It is an error to develop a study backward from data that has already been analyzed. For a data set or set of information, multiple statistical imputations or evaluations can be applied with eventual results that support a potential conclusion. This process is termed "data dredging" and is inappropriate in scientific research as it leads to flawed and biased conclusions that are the results of subjective manipulation of data as opposed to objective observation and analysis of data. While it seems obvious, it is important to stress that a research objective is developed to form the basis for a study, which is then conducted. The statement of the research objective (hypothesis) determines the types of study methodology and data that are appropriate to answer the research question. ${ }^{2}$

\section{The Well-designed Research Objective Statement}

To evaluate a research objective statement, it is convenient to consider a checklist known as the PICOT Criteria. ${ }^{2}$ Depending on the intent of the research, the PICOT Criteria summarize the elements needed for a well-stated research objective (Table 1). For all research objective statements, it is necessary to state who or what the study population will be. It is also required that the intervention for the study be declared. For prehospital and disaster 
research, the intervention may be an event, a change in practice or policy, a simple evaluation, or other action applied to the study population. A comparison group for a population that has had an intervention applied is usually necessary to determine if an outcome of interest changed or occurred. A time element for the length of research or follow-up of an intervention, or simply stating the dates or the period of study, is preferred in the research statement.

\section{The Research Objective Statement and a Hypothesis} Statement

A well-developed research objective statement provides the basis for a study, including the study population, applicable interventions and comparisons and the outcome of interest for a period of time. A hypothesis is a statement of or explanation of presumed facts that is made in such a way that the statement can be investigated or tested. For most prehospital and disaster health and medical studies, a concise research objective statement is equivalent to a hypothesis.

References

[1] Stratton SJ. Is there a scientific basis for disaster health and medicine? Prehosp Disaster Med. 2014;29(3):221-222.

[2] Brian HR. Forming research questions. J Clin Epidemiol. 2006;59(9):881-886.

While controversial, for a study in which there is testing of statistical significance, it is customary to state the hypothesis as a "null" hypothesis. A null hypothesis states that a research intervention or comparison results in no difference in outcome. As an approach to significance testing, the null hypothesis is statistically evaluated to determine if it can be discarded for any plausible alternative hypothesis. The alternative hypothesis is usually the outcome of interest for the research objective and accepted if the null hypothesis is discarded and shown not to be true.

\section{Summary}

A concise, well-designed research objective statement for proposed research is important for directing and coordinating a study. While often only a single or a few sentences in a manuscript, the research objective statement is usually the single most important element in the design and conduct of a research study.

doi:10.1017/S1049023X14000818 